\title{
Research on Inference Engine of Vehicle Fault Diagnosis Expert System Zhiyu Huang ${ }^{1}$, Xi Peng ${ }^{2}$ \\ ${ }^{1}$ School of Chongqing University of Posts and Telecommunications, Chongqing, 400065, China \\ ${ }^{2}$ School of Chongqing University of Posts and Telecommunications, Chongqing, 400065, China
}

Keywords: optimized inference engine, expert system, vehicle, fault diagnosis.

\begin{abstract}
To solve the problem of efficient reasoning and self-learning ability in expert system containing production representation knowledge, paper proposed an approach, sort-selection algorithm, to optimize the process of sort and, updated the confidence of all the knowledge according to frequency of the selection user chooses to realize the self-learning ability for expert system. Paper discussed the theory and process of the approach and applied it to the Vehicle Fault Diagnose Expert System. The testing result shows that these approaches could be easily realized and improved the efficiency of vehicle fault diagnosis.
\end{abstract}

\section{Introduction}

Inference engine, an indispensable part of knowledge system, includes reasoning and control and realizes the reasoning based on knowledge on the computer. In order to improve the efficiency and accuracy of reasoning in expert system, the reasoning strategy of inference engine depends on the expression way of diagnosis knowledge[1].

By comparing and analysing the theory and process of the inference engine in expert system, mainly for the production representation knowledge, paper figured out that it's really easy to improve the efficiency of reasoning if we optimize the sorting process of inference engine. Sorting by the knowledge confidence, choosing the maximum or minimum level of knowledge confidence, if the knowledge is required, the reasoning will end, or not, it will stop to sort the rest of knowledge through confidence again to select the maximum or minimum of knowledge, and that cycle repeats, until finding the matched knowledge at last, if the last filter to find matched knowledge, reasoning machine will quit.

Knowledge base is composed of specific knowledge recording opinions and thoughts form experts in particular field, they have had the subjectivity property in a way ,and this is due to the fact that each and every of experts has different knowledge and thoughts, therefore, knowledge base must constantly update and modify errors knowledge in practice[2].

The self-learning algorithm can be realized easily, and it increases or reduces the confidence of knowledge depending on the frequency of certain knowledge user invoking.

\section{The Optimized Reasoning}

Typical inference engine sorts all conclusions one-time when sorting reasoning conclusion set[3-4], such as vehicle fault diagnosis expert system based on the android OS. 


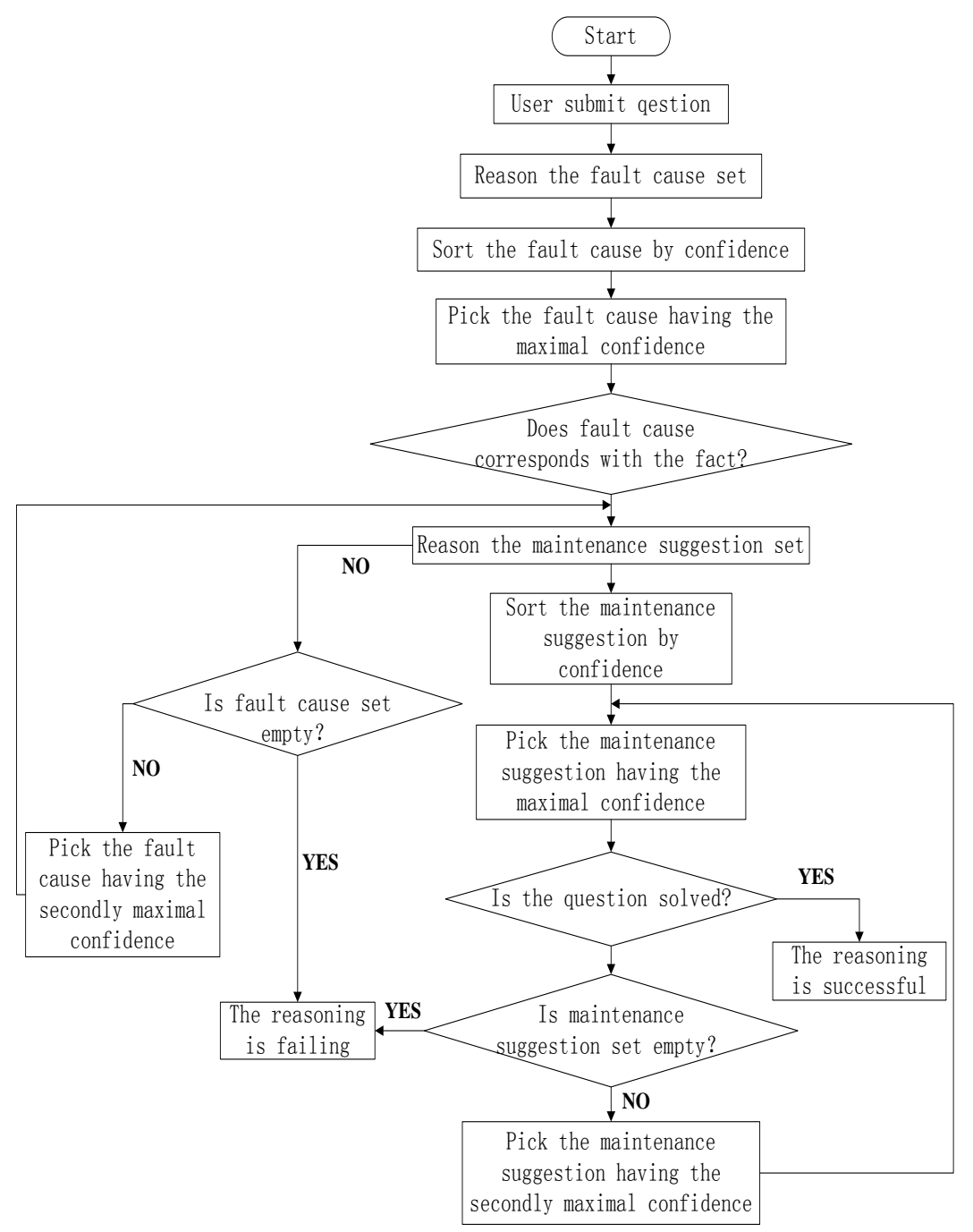

Fig. 1 The typical inference engine flowchart

The first step is reasoning out of all the fault causes depending on what the user input fault, and sort all causes in one-time by the confidence of every fault causes, then reason out the all corresponding maintenance suggestion set according to a malfunction reason that the user selected, next sort all maintenance suggestion in one-time by the confidence. The flow chart is shown in Fig.1[5].

It can be found from the chart, the inference engine needs twice sorting in total, if a fault is caused by $\mathrm{m}$ fault causes, and every fault cause correspond to $\mathrm{n}$ maintenance suggestions, inference engine need to sort $\mathrm{m}$ fault causes at first, and then sort $\mathrm{n}$ maintenance suggestions.

Duo to that the fault causes and maintenance suggestions have been sorted by confidence, as a result, the largest confidence of fault cause might have located the fault yet, the largest confidence of maintenance suggestion might guide the user to complete the work of vehicle maintenance, and the remaining sorted fault causes and maintenance suggestions serves no purpose. Based on the above consideration, paper analyzes the characteristics of the various sorting algorithms, and selection sort algorithm is adopted as the sorting algorithm of inference engine.

The thought of sort- algorithm is to identify the maximum or minimum element from the unsorted sequence, and put it at the end of a sorted sequence in order, moving in cycles until the unsorted sequence is empty. For example, the confidence set which the fault cause sequence correspond to is: $\{0.2,0.5,0.9,0.4,0.6\}$, the process of selection sort algorithm is as follows:

The first round: $\{0.9\}\{0.2,0.5,0.4,0.6\}$

The second round: $\{0.9,0.6\}\{0.2,0.5,0.4\}$

The third round: $\{0.9,0.6,0.5\}\{0.2,0.4\}$

The fourth round: $\{0.9,0.6,0.5,0.4\}\{0.2\}$ 
The last round: $\{0.9,0.6,0.5,0.4,0.2\}$

The reasoning flow chart is shown in Fig.2:

Because the selection sort algorithm takes out the largest confidence element from unsorted sequence, and the element is most likely to help user locate the fault causes and offer corresponding maintenance suggestions. For example, if user has used the fault cause got from the first round to locate the vehicle cause, the rest of the fault cause set doesn't need sorting, for the same reason, if user solve the vehicle cause with the maintenance suggestion in the first round, the rest of the maintenance suggestion set doesn't need sorting.

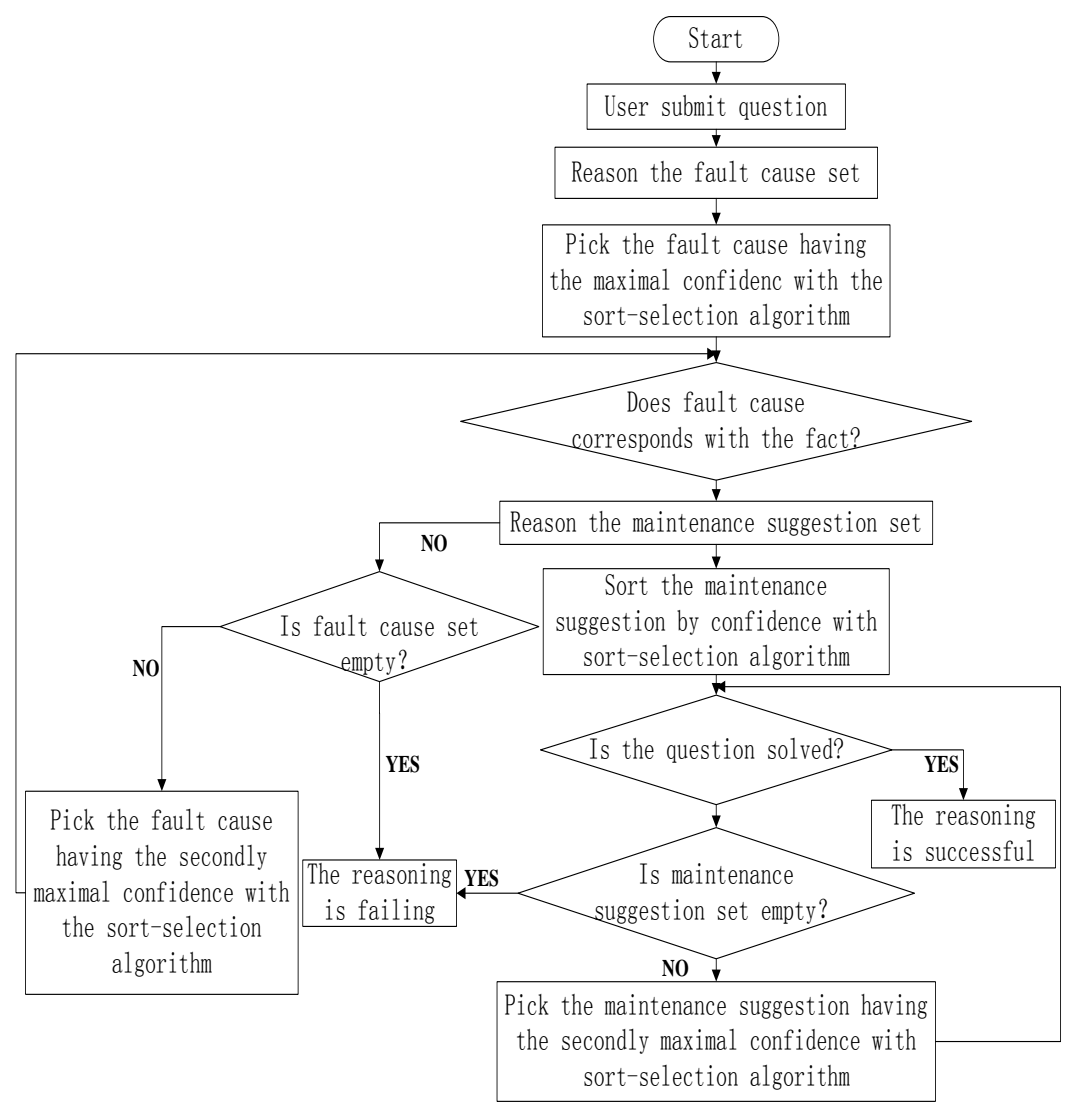

Fig.2 the inference engine flowchart with sort-selection algorithm

\section{The ability of self-learning}

First of all, the development of expert system begins with the establishment of knowledge base, knowledge base is to store the knowledge of expert system, and the process of establishing knowledge base is mainly by recording the understanding of each expert[5-6].

Because of the difference of each expert's experience and knowledge, the knowledge have had a subjective to a certain extent[7], therefore it must be updated and modified in practice constantly. As in the knowledge base of the Vehicle Fault Diagnose Expert System, the confidence of each fault causes and maintenance advices are much subjective, expert system needs to modify corresponding confidence dynamically according to the confirmation frequency of each fault cause and maintenance advice for user during the operation.

The flow chart of self-learning algorithm is shown in Fig.3. 


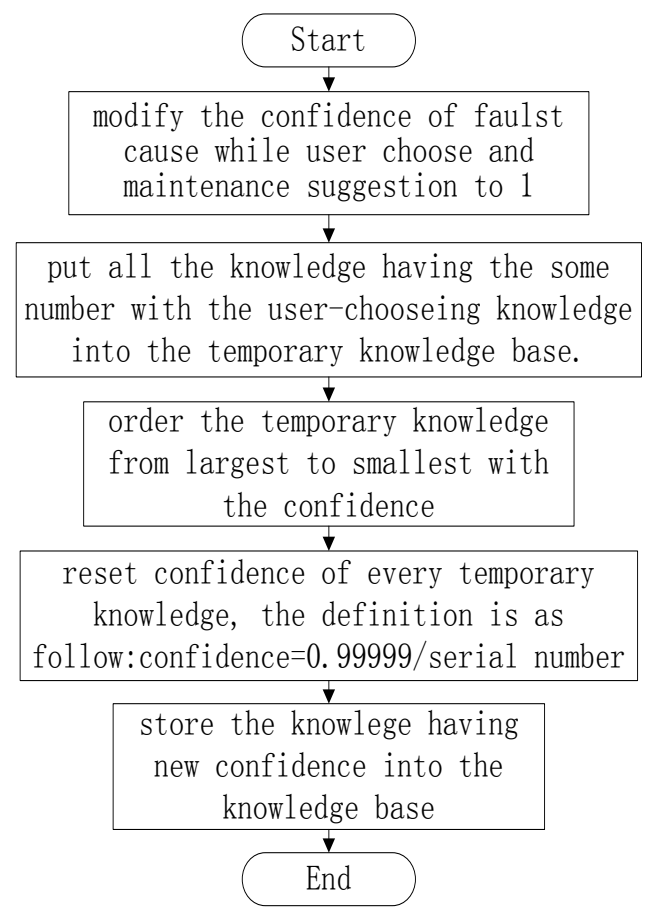

Fig.3 The self-learning flowchart

Taking fault, "the battery is over voltage", for example in the original knowledge base, there have three fault causes in statistics, their confidence and names are listed as follows.

0.8 "micro-short circuit inside batteries or short circuit between batteries"

0.7 "electric leakage in the power system"

0.6 "short circuit within the battery pack"

Adding the self-learning algorithm into the expert system, according to the continual user's inputs and the continual user's enquires, expert system could update and correct the level of confidence of any knowledge in dynamic.

\section{The testing result}

When user acknowledges that a certain conclusion corresponds with the fact, user click the right button, then expert system leads to maintenance-suggestion-reasoning interface.

As shown on the left in Figure 4, if user resolve the vehicle fault with the maintenance suggestion, expert system will leads to the interface shown on the middle in Figure 4, if not, the inference engine will select the highest confidence of knowledge from the remaining, if all maintenance suggestion are not direct users to troubleshoot, the expert system will leads to the interface shown on the right in Figure 4. All the tests show that those algorithms improve the efficiency and accuracy. 


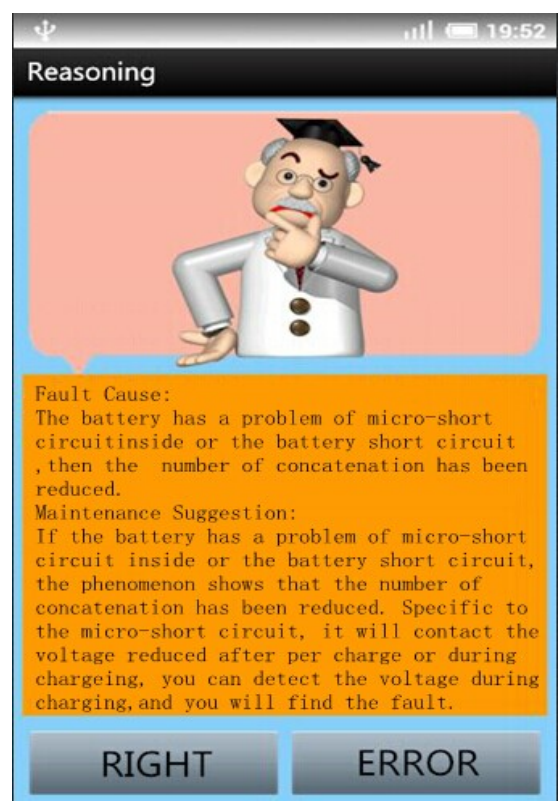

t) unl that 19:52

\section{Reasoning}

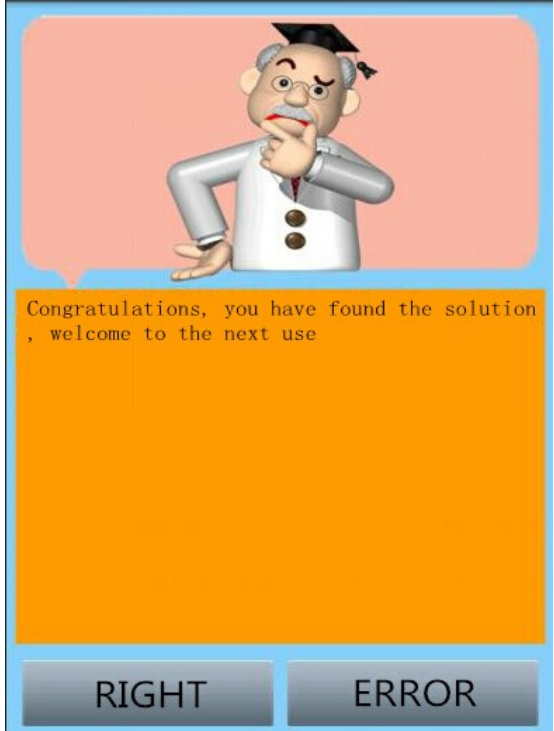

t) utl 대 19:52

\section{Reasoning}

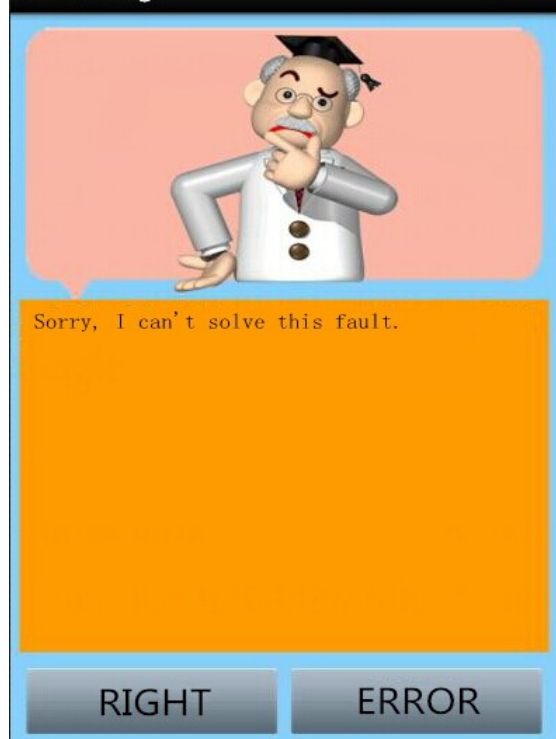

Fig.4 Result of reasoning 


\section{Conclusions}

Paper proposed the sort-selection algorithm and the self-learning algorithm for the sake of veracity and efficiency of reasoning in the inference engine. The sort-selection algorithm picked the knowledge having the maximum confidence to avoid sorting all the knowledge, and saved time and improved efficiency during the reasoning. The self-learning algorithm increased or increased the confidence of knowledge by means of times that user called the knowledge to realize the automatic update of knowledge and avoided the subjectivity of knowledge. After testing the two main algorithms in Vehicle Fault Diagnose expert system, it is much easy to realize the two algorithms, and it is also much obvious to improve the efficiency and efficiency.

\section{Acknowledgements}

The research was financially supported by the Chongqing Municipal Education Commission of Science and Technology Research Project (KJ120511) and Special Foundation for the Project of Internet of Things.

\section{References}

[1] WenBin,C., Xiaoling,L., Yu, F., \& Jian,Z. Inference Engine Design of Expert System Based on Blackboard Model and Fault Tree. Asia-Pacific Conference on Information Processing,1,pp.1820,2009.

[2] Yan Yang, The design and implementation of aeroengine fault diagnosis expert system knowledge base, Shenyang Aerospace University, 2012.

[3] Guicheng,W., Xue,W., Zhixin,R., Min,Z., Zhansheng,Z., \& Jinna,L. Study of fault diagnoses expert system of glutamic acid fermentation process. In Control and Decision Conference (CCDC),pp.3742-3747,2012.

[4] Cui,J., Wang,Y., Li,Z., Liu,L., \& Xu,G. A health diagnosis method for aeroplane structural component based on fault inference engine. Control and Decision Conference (CCDC),pp.3928-3931,2010.IEEE.

[5] YaLan W., The Research and Application on Inference Engine in Expert System. Wuhan University of Technology, pp.29-35,2006.

[6] Liu,X., \& Zhou,B. Research on knowledge base of dam safety monitoring expert system based on relational database. Knowledge Acquisition and Modeling Workshop,pp.11131116,2008.IEEE.

[7] WenBin,C., Xiao-Ling,L., ChangJiu,H., \& YiJun,L. Knowledge base design for fault diagnosis expert system based on production rule. Asia-Pacific Conference on Information Processing, Vol.1,pp.117-119,2009.

[8] Information on http://www.elecfans.com/lab/ceshiceliang/2009070675308.htMl 